\title{
Adaptive high-order splitting methods for systems of nonlinear evolution equations with periodic boundary conditions
}

\author{
Winfried Auzinger ${ }^{1}$. Othmar Koch ${ }^{2}$. \\ Michael Quell ${ }^{1}$
}

Received: 23 December 2015 / Accepted: 6 September 2016 / Published online: 4 October 2016 (C) The Author(s) 2016. This article is published with open access at Springerlink.com

\begin{abstract}
We assess the applicability and efficiency of time-adaptive high-order splitting methods applied for the numerical solution of (systems of) nonlinear parabolic problems under periodic boundary conditions. We discuss in particular several applications generating intricate patterns and displaying nonsmooth solution dynamics. First, we give a general error analysis for splitting methods for parabolic problems under periodic boundary conditions and derive the necessary smoothness requirements on the exact solution in particular for the Gray-Scott equation and the Van der Pol equation. Numerical examples demonstrate the convergence of the methods and serve to compare the efficiency of different time-adaptive splitting schemes and of splitting into either two or three operators, based on appropriately constructed a posteriori local error estimators.
\end{abstract}

Keywords Nonlinear evolution equations $\cdot$ Splitting methods $\cdot$ Adaptive time integration - Local error · Convergence

Mathematics Subject Classification (2010) $65 \mathrm{~J} 10 \cdot 65 \mathrm{~L} 05 \cdot 65 \mathrm{M} 12 \cdot 65 \mathrm{M} 15$

Winfried Auzinger

w.auzinger@tuwien.ac.at

Othmar Koch

othmar@othmar-koch.org

Michael Quell

michael.quell@yahoo.de

1 Institut für Analysis und Scientific Computing, Technische Universität Wien, Wiedner Hauptstraße 8-10/E101, 1040 Wien, Austria

2 Fakultät für Mathematik, Universität Wien, Oskar-Morgenstern-Platz 1, 1090 Wien, Austria 


\section{Introduction}

We are interested in computational methods for nonlinear evolution equations of the type

$$
\partial_{t} u(t)=A u(t)+B(u(t)), \quad t>t_{0},
$$

on a Banach space $\mathcal{B}$, which in our examples equals $L^{2}$ on the $d$-dimensional torus. Here, $A: \mathcal{D} \subseteq \mathcal{B} \rightarrow \mathcal{B}$ is an (unbounded) differential operator and $B$ a generally an unbounded nonlinear operator whose domain has nonempty intersection with $\mathcal{D}$.

To enable an efficient numerical solution of (1.1) for large-scale applications, adaptive high-order time-discretizations are central. In some applications, the promised speed-up will be critical for the feasibility of a simulation. In many realistic models, the stiffness of the operators $A$ and $B$ is different, which suggests to use splitting methods which separately propagate the two vector fields. If $A$ is a linear differential operator, effective schemes are known which solve the subproblem efficiently after appropriate space discretization. For the problems discussed in this paper, a Fourier pseudospectral space discretization is the most natural choice as this allows to propagate the linear part by exponentiation of a diagonal matrix.

Parabolic equations often induce high computational demand due to challenging solution dynamics, which suggests to employ adaptive time-stepping in order to accommodate for local variations in the numerical error. However, this is not the only reason for using adaptivity. Typically, the optimal step-size is not known a priori, and an adaptive procedure determines the appropriate value within a few steps, see for example Section 3.3. Moreover, adaptive time-stepping increases the reliability of a computation, see for instance [1].

At the (time-)semi-discrete level, $s$-stage exponential splitting methods for the integration of (1.1) use multiplicative combinations of the partial flows $\phi_{A}(t, u)$ and $\phi_{B}(t, u)$. For a single-step $\left(0, u_{0}\right) \mapsto\left(h, u_{1}\right)$ with time-step $t=h$, this reads

$$
u_{1}:=\mathcal{S}\left(h, u_{0}\right)=\phi_{B}\left(b_{s} h, \cdot\right) \circ \phi_{A}\left(a_{s} h, \cdot\right) \circ \ldots \circ \phi_{B}\left(b_{1} h, \cdot\right) \circ \phi_{A}\left(a_{1} h, u_{0}\right),
$$

where the coefficients $a_{j}, b_{j}, j=1 \ldots s$ are determined according to the requirement that a prescribed order of consistency is obtained [2].

Compared to highly implicit methods as for instance implicit Runge-Kutta methods or their exponential counterparts (see [3]), splitting methods are easy to implement and efficient in combination with suitable spatial discretization and appropriate implementations or approximations of the subflows $\phi_{A}$ and $\phi_{B}$. This is an important asset of our approach; however, we will demonstrate in addition that adaptive choice of the time-steps leads to a more efficient solution for problems where the variation in the solution is large. For related work on adaptivity using a pair of lower order methods, we refer to [4].

A rigorous error analysis of splitting methods for Schrödinger equations has first been given for the second-order Strang splitting scheme in [5], which has later been extended to higher-order splittings in [6]. The more involved arguments for the nonlinear case have been devised in [7] for the Schrödinger-Poisson and cubic nonlinear 
Schrödinger equation for second-order splitting; higher-order methods are analyzed in [8].

The error analysis relies on an error representation which was first proven in [8]: the local error of a splitting method of order $p$ applied to a nonlinear evolution equation has an error expansion with leading term

$$
\mathcal{L}(h, u) \sim \sum_{k=1}^{p} \sum_{\substack{\mu \in \mathbb{N}^{k} \\|\mu| \leq p-k}} \frac{1}{\mu !} h^{k+|\mu|} C_{k \mu} \prod_{\ell=1}^{k} \operatorname{ad}_{D_{A}}^{\mu_{\ell}}\left(D_{B}\right) \mathrm{e}^{h D_{A}} u,
$$

where $C_{k \mu}$ are computable constants and $D_{A}, D_{B}$ represent the Lie derivatives of the two vector fields, respectively. $\operatorname{ad}_{D_{A}}^{\mu_{\ell}}\left(D_{B}\right)$ denotes the $\mu_{\ell}$-fold commutator. In our subsequent analysis, we will make use of this error representation, where the main task will be to compute and estimate the commutators of the vector fields in an appropriate functional analytic setting in the space of periodic functions. To this end, we will resort to a Sobolev theory on the torus, which we review in detail in Appendix A, to which we refer for notations used in the subsequent error analysis.

Detailed understanding and analysis of splitting methods for parabolic problems in particular for the nonlinear case is missing to date. Partial results have been obtained by other authors; recent work for linear problems can be found in [9] and [10]. In particular, in [9], a number of higher-order methods with complex coefficients are constructed. In these papers, splitting methods are analyzed in the context of semigroup theory. However, the authors do not exploit the special structure of the local error (as specified in [11] in terms of iterated commutators). Therefore, the results in [10] rely on unnaturally restrictive regularity assumptions, and the same is true for the convergence results given in [9].

Section 2 introduces a number of local a posteriori error estimators whose performance will subsequently be assessed.

In Section 3, our theoretical framework is applied to analyze the convergence of splitting methods for the Gray-Scott equation, where the regularity requirements on the exact solution are worked out which ensure boundedness of the commutators appearing in the error expansion.

In Section 4, we investigate the Van der Pol system, which has a stiff limit cycle. Adaptive time-stepping is shown to give rise to guaranteed accuracy, and in some cases significantly reduced computation times compared to fixed time-steps.

In Section 5, we demonstrate that splitting into three operators can be beneficial computationally if the structure of the vector field enables exact integration of the subproblems, by resorting to computations for the Gray-Scott equations.

The functional analytic framework for the error analysis of splitting methods applied to parabolic problems under periodic boundary conditions is briefly recapitulated in Appendix A, which states the underlying results for the space of periodic functions on the torus. Sobolev embeddings which are used in our error estimates are stated in Appendix B with a brief indication of the proofs. 


\section{A posteriori local error estimators}

In this section, we briefly describe three classes of computable a posteriori local error estimators which serve as our basis for adaptive time-stepping and which have different advantages depending on the context in which they are applied. Embedded pairs of splitting formulae have been introduced in [12] and are based on reusing a number of evaluations from the basic integrator. For methods of odd order, an asymptotically correct error estimator can be computed at the same cost as for the basic method by employing the adjoint method, see [14], and finally the Milne device relies on the explicit knowledge of the leading error terms of methods of equal order. A collection of splitting coefficients covering also these three types of error estimators has been compiled at the webpage

http://www.asc.tuwien.ac.at/ winfried/splitting/

which we subsequently refer to as [15].

\subsection{Embedded pairs}

In [12], pairs of splitting schemes of orders $p$ and $p+1$ are specified. The idea is to select a controller $\overline{\mathcal{S}}$ of order $p+1$ and to construct an integrator $\mathcal{S}$ of order $p$ for which a maximal number of compositions coincide with those of the controller. To construct pairs offering an optimal balance between cost and accuracy, we fix a "good" controller of order $p+1$ and wish to adjoin to it a "good" integrator of order $p$. Since the number of compositions $\bar{s}$ in the controller will be higher than the number of compositions $s$ in the integrator, we can select an optimal embedded integrator $\mathcal{S}$ from a set of candidates obtained by flexible embedding, where the number of coinciding coefficients is not a priori fixed. The idea is expanded in detail in [14], where optimized methods are determined.

\subsection{Adjoint pairs and palindromic formulae}

For a scheme $\mathcal{S}$ of odd order $p$, the leading local error terms of $\mathcal{S}$ and its adjoint $\mathcal{S}^{*}$ are identical up to the factor -1 , see [2]. Therefore, the averaged additive scheme

$$
\overline{\mathcal{S}}(h, u)=\frac{1}{2}\left(\mathcal{S}(h, u)+\mathcal{S}^{*}(h, u)\right)
$$

is a method of order $p+1$, and

$$
\mathcal{P}(h, u):=\mathcal{S}(h, u)-\overline{\mathcal{S}}(h, u)=\frac{1}{2}\left(\mathcal{S}(h, u)-\mathcal{S}^{*}(h, u)\right)
$$

provides an asymptotically correct local error estimate for $\mathcal{S}(h, u)$. In this case, the additional effort for computing the local error estimate is identical with the effort for the integrator $\mathcal{S}$ but not higher as is the case for embedded pairs. This principle is limited to methods of odd order. In particular, in [14], so-called palindromic schemes were constructed which turn out to have small error constants as compared to competing schemes. Therefore, we include palindromic pairs in our investigations. 


\subsection{The Milne device}

In the context of multi-step methods for ODEs, the so-called Milne device is a wellestablished technique for constructing pairs of schemes. In our context, one may aim for finding a pair $(\mathcal{S}, \tilde{\mathcal{S}})$ of schemes of equal order $p$ such that their local errors $\mathcal{L}, \tilde{\mathcal{L}}$ are related according to

$$
\begin{aligned}
& \mathcal{L}(h, u)=C(u) h^{p+1}+\mathscr{O}\left(h^{p+2}\right), \\
& \tilde{\mathcal{L}}(h, u)=\gamma C(u) h^{p+1}+\mathscr{O}\left(h^{p+2}\right),
\end{aligned}
$$

with $\gamma \neq 1$. Then, the additive scheme

$$
\overline{\mathcal{S}}(h, u)=-\frac{\gamma}{1-\gamma} \mathcal{S}(h, u)+\frac{1}{1-\gamma} \tilde{\mathcal{S}}(h, u)
$$

is a method of order $p+1$, and

$$
\mathcal{P}(h, u):=\mathcal{S}(h, u)-\overline{\mathcal{S}}(h, u)=\frac{1}{1-\gamma}(\mathcal{S}(h, u)-\tilde{\mathcal{S}}(h, u))
$$

provides an asymptotically correct local error estimate for $\mathcal{S}(h, u)$.

\subsection{Step-size selection}

Based on a local error estimator, the step-size is adapted such that a prescribed local error tolerance tol is expected to be satisfied in the subsequent step. If $h_{\text {old }}$ denotes the current step-size, the next step-size $h_{\text {new }}$ is predicted as (see $[16,17]$ )

$$
h_{\text {new }}=h \cdot \min \left\{\alpha_{\text {max }}, \max \left\{\alpha_{\text {min }},\left(\alpha \frac{\text { tol }}{\mathcal{P}\left(h_{\text {old }}\right)}\right)^{\frac{1}{p+1}}\right\}\right\} \text {, }
$$

where we choose $\alpha=0.9, \alpha_{\min }=0.25, \alpha_{\max }=4.0$. This simple strategy incorporates safety factors to avoid an oscillating and unstable behavior. The chosen values of $\alpha_{\min }$ and $\alpha_{\max }$ are commensurable with the recommendations in [2]. The safety factors have not proven critical in our examples; the local changes in the step-sizes are usually smaller from step to step, see for example Fig. 8. Only if at the beginning of time propagation the initial step-size is unsuitable as in Fig. 6, where still no instabilities arise in the step-size control, however.

\section{The Gray-Scott equation}

As a concrete example, we first study the Gray-Scott system (see [18]) modeling a two-component reaction-diffusion process,

$$
\begin{aligned}
& \partial_{t} u(x, y, t)=c_{u} \Delta u(x, y, t)-u(x, y, t) v^{2}(x, y, t)+\alpha(1-u(x, y, t)), \\
& \partial_{t} v(x, y, t)=c_{v} \Delta v(x, y, t)+u(x, y, t) v^{2}(x, y, t)-\beta v(x, y, t) .
\end{aligned}
$$

This system is of the type (1.1), with unknown $(u(x, y, t), v(x, y, t))$, the vector of concentrations of the two chemical species involved. In many situations, this model is closed naturally by periodic boundary conditions. This system is studied as a model 

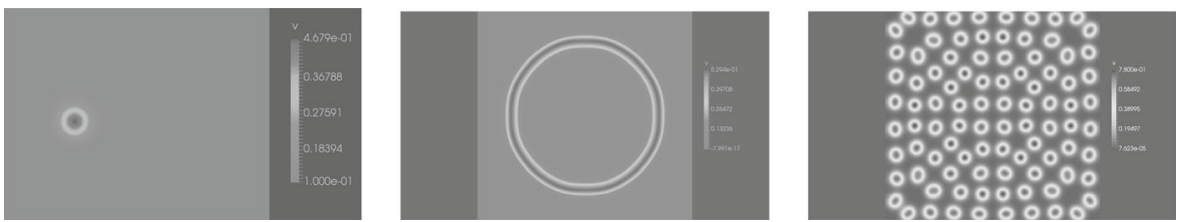

Fig. 1 Solution component $v$ at $t=0$ (left), $t=2000$ (middle), and $t=4000$ (right) for (3)

for pattern formation with a rich dynamical behavior. For $(x, y) \in[-4 \pi, 4 \pi]^{2}$, we prescribe the initial condition

$u(x, y, 0)=0.5+\exp \left(-1-\left(x^{2}+y^{2}\right)\right), \quad v(x, y, 0)=0.1+\exp \left(-1-\left(x^{2}+y^{2}\right)\right)$.

A visualization of the solution component $v$ at $t=0,2000$ and 4000 is shown in Fig. 1.

The problem can also naturally be stated in three spatial dimensions and solved by our methods. In Fig. 2, we show the component $v$ computed by a complex embedded $4 / 3$ splitting pair from [12] with an underlying spatial discretization with $512^{3}$ basis
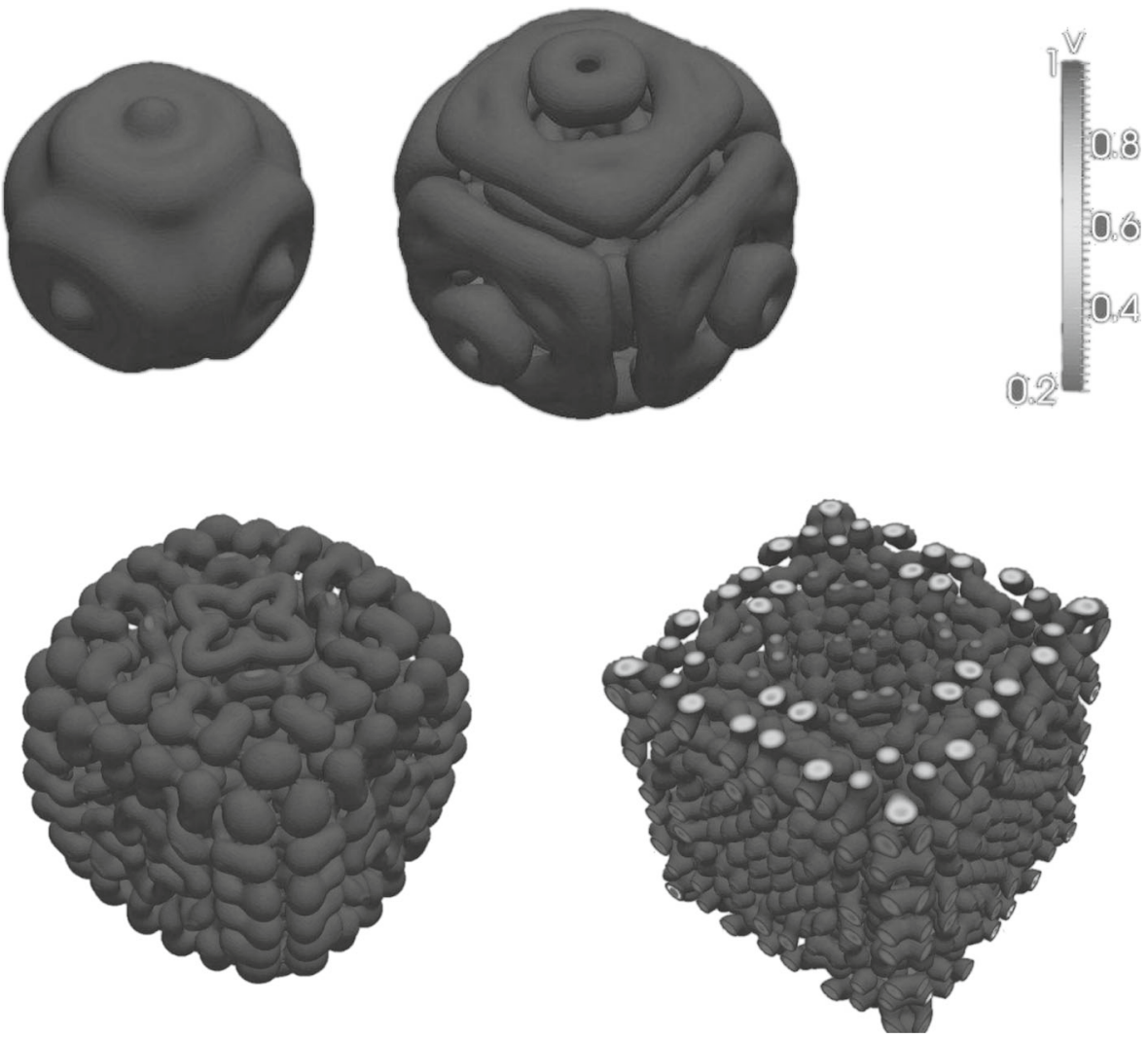

Fig. 2 Solution component $v$ for (3) in 3D at times $t=2500,3000,4000,5000$ 
functions and a tolerance of $10^{-5}$. The solution is plotted at times $t=2500, t=$ $3000, t=4000$, and $t=5000$. In the following, we will only investigate the $2 \mathrm{D}$ case, as this does not influence the assessment of the time integrators, but reduces computation time.

\subsection{Convergence analysis}

For the theoretical analysis of the convergence of splitting methods, we use the error representation (1.3). Since the flow induced by the cubic nonlinearity is not unconditionally stable, we have to resort to the three-stage argument first given in [7] for the cubic Schrödinger equation, see also [8]:

- First, show stability in the $H^{2}$-norm.

- The local error in $H^{2}$ is $O\left(h^{p-1}\right)$, where the constant depends on the $H^{2 p-2}$ norm of $u$.

- Stability together with consistency in $H^{2}$ implies convergence of order $p-2$ in $H^{2}$.

- Convergence implies boundedness of the numerical solution in $H^{2}$.

- Analyze stability in $H^{1}$. It turns out that the constant depends on the $H^{2}$ norms of both the exact and the numerical solution. The latter has already been demonstrated to be bounded.

- The local error in $H^{1}$ is $O\left(h^{p}\right)$, where the constant depends on the $H^{2 p-1}$-norm of $u$.

- Since $\left\|u_{n}\right\|_{H^{2}}$ is bounded, stability and consistency imply convergence order $p-1$ in $H^{1}$.

- Analyze stability in $L^{2}$. It turns out that the constant depends on the $H^{2}$ norms of both the exact and the numerical solution. The latter has already been demonstrated to be bounded.

- The local error in $L^{2}$ is $O\left(h^{p+1}\right)$, where the constant depends on the $H^{2 p}$-norm of $u$.

- We conclude convergence of order $p$ in $L^{2}$.

Along this line, we can prove the following theorem, since for the present situation of a parabolic problem under periodic boundary conditions, the same Sobolev embeddings hold as on the full space $\mathbb{R}^{3}$, see Appendix A, so in particular the second order differential operators and the cubic terms and their commutators admit the same bounds. Thus, the following proof strategy can be followed in the same manner, taking into account the commutator bounds given later:

Theorem 1 Suppose that the Gray-Scott (3) possesses a uniquely determined sufficiently regular solution $u$ on the time interval $[0, T]$. Then, for any exponential operator splitting method (1.2) of (nonstiff) order $p \geq 2$, the following error estimates are valid.

1. Provided that $\|u(t)\|_{H^{2 p}} \leq M_{2 p}$ for $0 \leq t \leq T$, the bound

$$
\left\|u_{n}-u\left(t_{n}\right)\right\|_{L^{2}} \leq C h^{p}, \quad 0 \leq n \leq N, \quad t_{N} \leq T
$$


holds true with constant $C$ depending on $M_{2 p}$.

2. Provided that $\|u(t)\|_{H^{2 p-1}} \leq M_{2 p-1}$ for $0 \leq t \leq T$, the bound

$$
\left\|u_{n}-u\left(t_{n}\right)\right\|_{H^{1}} \leq C h^{p-1}, \quad 0 \leq n \leq N, \quad t_{N} \leq T,
$$

holds true with constant $C$ depending on $M_{2 p-1}$.

3. Provided that $\|u(t)\|_{H^{2 p-2}} \leq M_{2 p-2}$ for $0 \leq t \leq T$, the bound

$$
\left\|u_{n}-u\left(t_{n}\right)\right\|_{H^{2}} \leq C h^{p-2}, \quad 0 \leq n \leq N, \quad t_{N} \leq T,
$$

holds true with constant $C$ depending on $M_{2 p-2}$.

Proof We work out the analysis in detail for the case $p=2$; the general case is proven analogously. For the analysis, we write the Gray-Scott system in the partitioned form

$$
\begin{aligned}
\partial_{t} U(x, y, t) & =A U(x, y, t)+B(U(x, y, t)), \\
U(x, y, 0) & =U_{0}(x, y), \quad(x, y) \in[-\pi, \pi]^{2},
\end{aligned}
$$

where

$$
\begin{aligned}
& U(x, y, t)=\left(\begin{array}{l}
u(x, y, t) \\
v(x, y, t)
\end{array}\right), \\
& A U(x, y, t)=\left(\begin{array}{cc}
c_{1} \Delta-\alpha & 0 \\
0 & c_{2} \Delta-\beta
\end{array}\right) U(x, y, t)+\left(\begin{array}{l}
\alpha \\
0
\end{array}\right), \\
& B(U(x, y, t))=\left(\begin{array}{c}
-u(x, y, t) v^{2}(x, y, t) \\
u(x, y, t) v^{2}(x, y, t)
\end{array}\right) .
\end{aligned}
$$

Stability is shown in the same manner as for the cubic Schrödinger equation [8], see the outline above. To bound the local error, we compute the commutators of the vector fields. This yields

$$
\begin{aligned}
{[A, B](U)=} & A B(U)-B^{\prime}(U) A U \\
= & \left(\begin{array}{cc}
c_{1} \Delta-\alpha & 0 \\
0 & c_{2} \Delta-\beta
\end{array}\right)\left(\begin{array}{c}
-u v^{2} \\
u v^{2}
\end{array}\right)+ \\
& +\left(\begin{array}{cc}
v^{2} & 2 u v \\
-v^{2}-2 u v
\end{array}\right)\left(\begin{array}{c}
\left(c_{1} \Delta-\alpha\right) u \\
\left(c_{2} \Delta-\beta\right) v
\end{array}\right) \\
= & \left(\begin{array}{c}
-c_{1} \Delta\left(u v^{2}\right)+v^{2} c_{1} \Delta u+2 u v\left(c_{2} \Delta v-\beta v\right) \\
\left(c_{2} \Delta-\beta\right) u v^{2}-v^{2}\left(c_{1} \Delta-\alpha\right) u-2 u v\left(c_{2} \Delta-\beta\right) v
\end{array}\right) \\
= & \left(\begin{array}{c}
2\left(c_{2}-c_{1}\right) u v \Delta v-4 c_{1} v \nabla u \cdot \nabla v-2 c_{1} u \nabla v \cdot \nabla v+2 c_{1} \beta u v^{2} \\
\left(c_{2}-c_{1}\right) v^{2} \Delta u+4 c_{2} v \nabla u \cdot \nabla v+2 c_{2} u \nabla v \cdot \nabla v+(\alpha+\beta) u v^{2}
\end{array}\right) .
\end{aligned}
$$

This can be estimated in Sobolev norms by resorting to the embeddings in Appendix B:

$$
\|[A, B](U)\|_{H^{m}} \leq C\left(\|U\|_{H^{m+2}}\right), \quad m=0,1, \ldots
$$


For the second commutator, we compute

$$
\begin{aligned}
& B^{\prime}(U) W=\left(\begin{array}{cc}
-v^{2} & -2 u v \\
v^{2} & 2 u v
\end{array}\right)\left(\begin{array}{l}
w_{1} \\
w_{2}
\end{array}\right) \\
& =\left(\begin{array}{c}
-v^{2} w_{1}-2 u v w_{2} \\
v^{2} w_{1}+2 u v w_{2}
\end{array}\right) \\
& B^{\prime \prime}(U)(W, Z)=\left(\begin{array}{cc}
0 & -2 u w_{2} \\
0 & 2 u w_{1}
\end{array}\right)\left(\begin{array}{l}
z_{1} \\
z_{2}
\end{array}\right) \\
& =\left(\begin{array}{c}
-2 u w_{2} z_{1} \\
2 u w_{1} z_{2}
\end{array}\right) \text {, } \\
& A^{2} U=\left(\begin{array}{c}
\left(c_{1} \Delta-\alpha\right)^{2} u \\
\left(c_{2} \Delta-\beta\right)^{2} v
\end{array}\right)+\left(\begin{array}{c}
\alpha\left(c_{1} \Delta-\alpha+1\right) \\
0
\end{array}\right)
\end{aligned}
$$

and hence

$$
[A,[A, B]](U)=A^{2} B(U)-2 A B^{\prime}(U) A U+B^{\prime \prime}(U)(A U, A U)+B^{\prime}(U) A^{2} U
$$

contains terms of the form $u v \Delta^{2} u$ and $u v \Delta^{2} v$ which do not cancel. Consequently,

$$
\|[A,[A, B]](U)\|_{H^{m}} \leq C\left(\|U\|_{H^{m+4}}\right), \quad m=0,1, \ldots
$$

Inductively, the result for higher commutators appearing in estimates for higher-order splitting methods follows.

\subsection{Numerical results}

In this section, we will demonstrate the accuracy of several splitting schemes for the Gray-Scott equation (3) by computing the convergence orders with an underlying Fourier pseudospectral space discretization at $512 \times 512$ points. The nonlinear terms in the equation are propagated using an explicit fourth-order RungeKutta method. For these experiments, the parameters in (3) were chosen as $\alpha=$ $0.038, \beta=0.114, c_{1}=0.04$, and $c_{2}=0.005$. We will investigate the pair [15, Milne $2 / 2 \mathrm{C}$ (i) ], and the optimized palindromic fourth-order method [15, Emb $4 / 3$ A c]. The error estimators are based on the Milne device (Section 2.3), and the embedding idea (Section 2.1), respectively. Figure 3 gives the error of the method [15, Milne 2/2 C (i)] and the error of the associated error estimator as well as the global error of the time integration. The empirical convergence order can be observed by comparing the computed data points with the solid line representing the theoretical order extrapolated from the most accurate approximation. Figure 4 gives the same data for the integrator from [15, Emb 4/3 A c] and associated error estimator. Errors are calculated with respect to a reference solution computed by [15, Emb $4 / 3 \mathrm{~A} \mathrm{C}]$ with time-step $h=7.81 \cdot 10^{-3}$. The empirical orders illustrate the theoretical result in Theorem 1 .

The time-steps generated in the course of an adaptive procedure are given in Fig. 5. The left plot shows the time-steps to satisfy a tolerance of $10^{-5}$ for the [15, Milne $2 / 2 \mathrm{C}(\mathrm{i})]$ method, and likewise on the right for the [15, Emb $4 / 3$ A c] pair. 


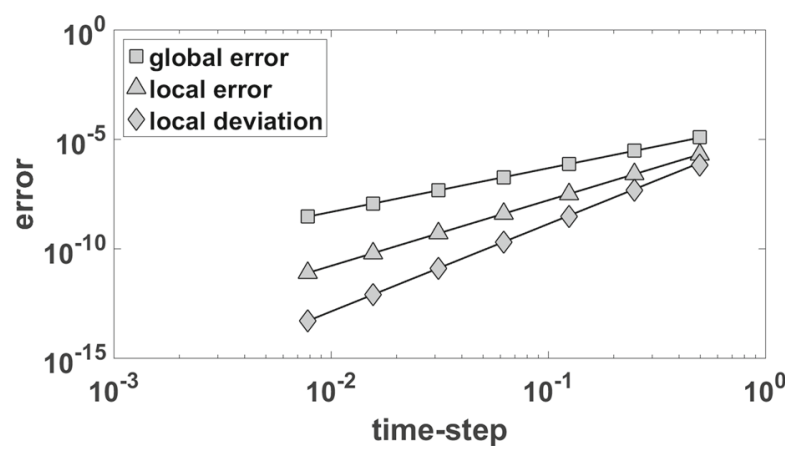

Fig. 3 Empirical convergence orders of the local and the global errors and deviation of the local error estimator for the [15, Milne $2 / 2$ C (i) ] splitting applied to the Gray-Scott (3)

\subsection{Comparisons}

After verifying the reliability of the investigated solution methods, we will assess the efficiency of the adaptive time integration methods by giving a comparison to the situation where the same accuracy is achieved with constant time-steps. Moreover, we will compare the efficiency of adaptive time integration based on the secondorder method in conjunction with the Milne device as compared to the fourthorder embedded splitting pair [15, Emb 4 (3) A c] and the palindromic scheme $[15, \mathrm{PP} 3 / 4 \mathrm{~A} \mathrm{C}]$. By construction, the latter also provides an asymptotically correct error estimator, which by its special structure is cheap to evaluate. Runtime was measured on a PC with Intel Core i7-2600 3, 4GHz Quad-Core processor with 16 GB RAM: Table 1 shows the number of steps required in the adaptive integration, the number of equidistant steps with the smallest necessary adaptive time-step, and the computing time for both scenarios. The tolerances were chosen as $10^{-5}$ (top) and $10^{-8}$ (bottom), respectively. We observe that indeed the adaptive methods require fewer steps, but the overall computational cost is higher due to the effort for the

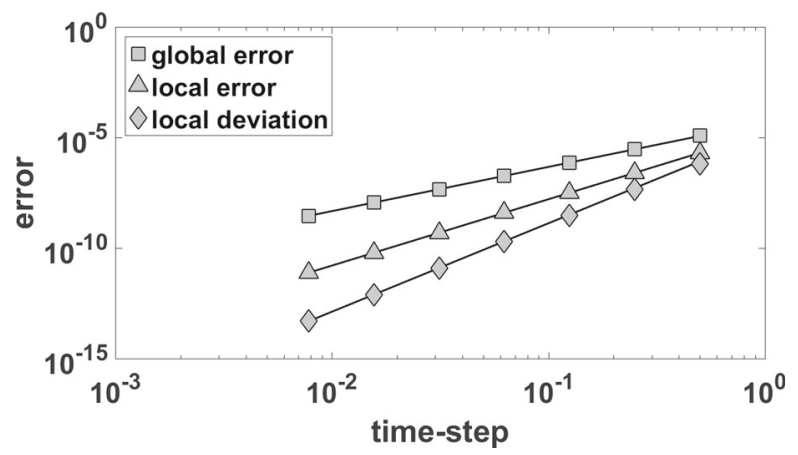

Fig. 4 Empirical convergence orders of the local and the global errors for the [15, Emb $4 / 3 \mathrm{~A} \mathrm{C}] \mathrm{splitting}$ and deviation of the local error estimator applied to the Gray-Scott (3) 

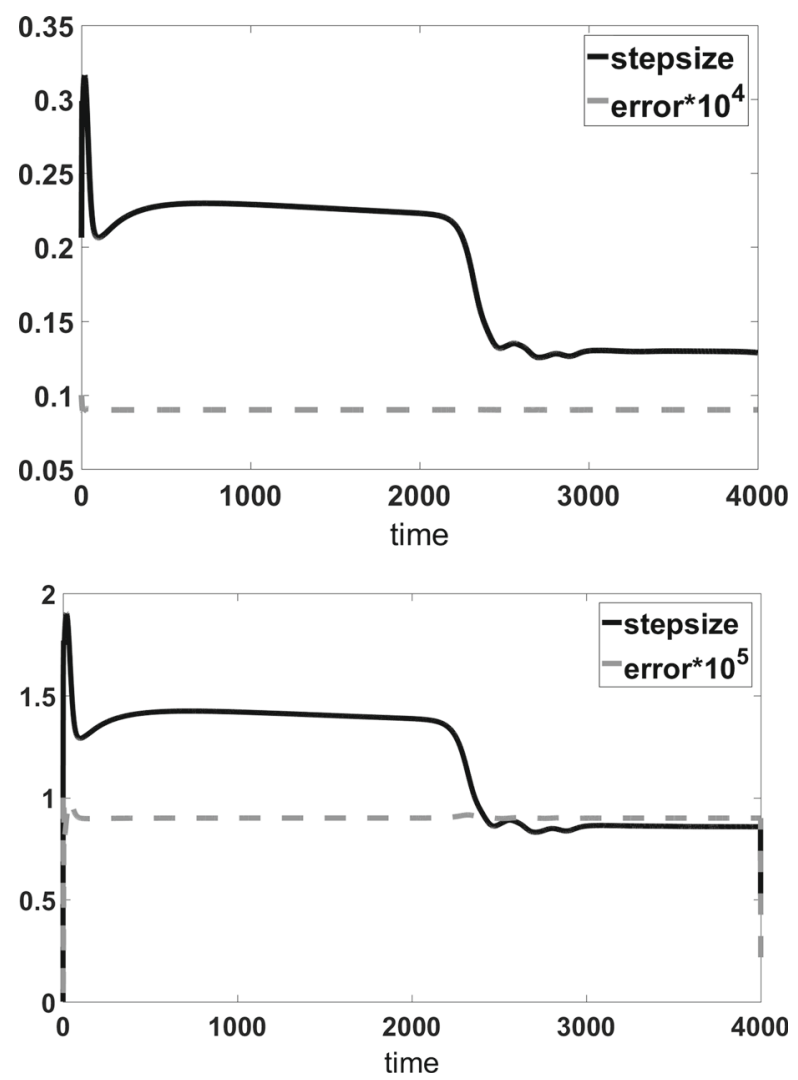

Fig. 5 Time-steps for (3) for [15, Milne 2/2 c (i) ] (top), [15, Emb $4 / 3$ A c] (bottom), tolerance $10^{-5}$

evaluation of the error estimator in each step. This suggests an adaptive strategy which does not estimate the error in each step, but only after a certain number of steps with a fixed time-step. This is also supported by the fact that a measurement of the computation time for the [15, Milne $2 / 2$ c (i) ] method on 1000 equidistant steps yielded $75.45 \mathrm{~s}$, in conjunction with the error estimator the computation time amounted to $123.43 \mathrm{~s}$. The same experiment for the [15, Emb $4 / 3 \mathrm{~A} \mathrm{c}]$ method yielded 162.18 and $238.36 \mathrm{~s}$, respectively. For [15, PP $3 / 4 \mathrm{~A} \mathrm{c}$, the run times were 110.75 and $193.68 \mathrm{~s}$, respectively. This implies that an update of the time-steps every two or three steps should provide a more efficient strategy, but possibly at the cost of reduced numerical stability, since this example shows rather smooth solution dynamics. Indeed, the step-size is adjusted rapidly by exploiting the maximally permitted increase by a factor of 4 from a too small initial guess to the appropriate value, which is assumed throughout the rest of the computation, see Fig. 6, which gives the quotient of two consecutive step-sizes over the integration interval. This behavior demonstrates one major advantage of adaptivity, that an unsuitable initial guess of the step-size is automatically adjusted to an optimal value. 
Table 1 Comparison of the efficiency of [15, Milne $2 / 2 \mathrm{C}$ (i)], [15, Emb 4/3 A c], and $[15, \mathrm{PP} 3 / 4 \mathrm{~A} \mathrm{C}]$ for (3)

\begin{tabular}{|c|c|c|c|c|}
\hline Method & \# Steps adaptive & \# Steps equidist & Time adaptive & Time equidist \\
\hline Milne $2 / 2 \mathrm{C}(\mathrm{i}), \mathrm{tol}=10^{-5}$ & 406 & 486 & 57.04 & 28.21 \\
\hline Emb $4 / 3 \mathrm{~A} \mathrm{C}$, tol $=10^{-5}$ & 67 & 79 & 17.72 & 11.46 \\
\hline $\mathrm{PP} 3 / 4 \mathrm{~A} \mathrm{C}$, tol $=10^{-5}$ & 116 & 135 & 23.02 & 12.99 \\
\hline Milne $2 / 2 \mathrm{C}(\mathrm{i}), \mathrm{tol}=10^{-8}$ & 4691 & 5625 & 878.72 & 503.93 \\
\hline Emb $4 / 3 \mathrm{~A} \mathrm{C}$, tol $=10^{-8}$ & 516 & 612 & 174.30 & 128.19 \\
\hline $\mathrm{PP} 3 / 4 \mathrm{~A} \mathrm{C}, \mathrm{tol}=10^{-8}$ & 929 & 1107 & 195.87 & 106.79 \\
\hline
\end{tabular}

The tolerances were $10^{-5}$ (top) and $10^{-8}$ (bottom), respectively

\section{The Van der Pol equation}

The Van der Pol equation is an ordinary differential equation with limit cycle behavior. It is used as a test of time integration schemes for stiff differential equations. It shares characteristics with simple models for cardiac behavior. The Van der Pol equation is usually considered as an ordinary differential equation, but by adding diffusion terms, one can consider an extension from a set of ordinary differential equations to a pair of coupled partial differential equations with spatial dependence.

It is given by

$$
\begin{aligned}
& \partial_{t} u(x, t)=D_{u} \Delta u(x, t)+v(x, t), \\
& \partial_{t} v(x, t)=D_{v} \Delta v(x, t)+\frac{1}{\epsilon}\left[\left(1-u^{2}(x, t)\right) v(x, t)-u(x, t)\right] .
\end{aligned}
$$

It is split into

$$
\begin{aligned}
& \partial_{t} u(x, t)=D_{u} \Delta u(x, t)+v(x, t), \\
& \partial_{t} v(x, t)=D_{v} \Delta v(x, t)+\frac{1}{\epsilon}(v(x, t)-u(x, t)),
\end{aligned}
$$

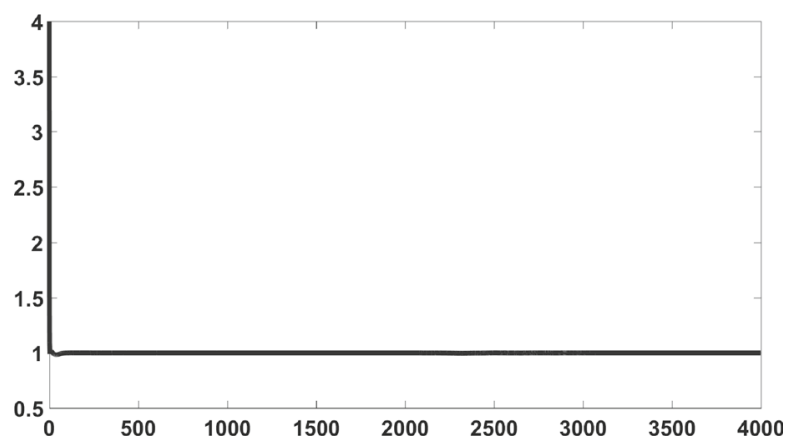

Fig. 6 Ratio of two consecutive time-step sizes for the solution of (3) by [15, Emb $4 / 3$ A c] 

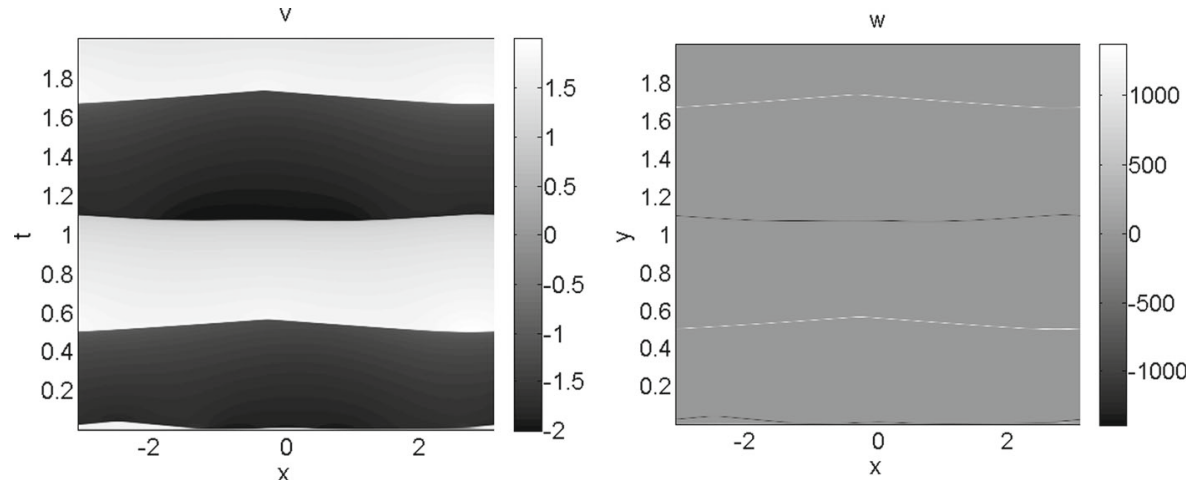

Fig. 7 Numerical solution for (4.1a) with $D_{u}=D_{v}=1$ and $\epsilon=10^{-3}, 256$ grid points, with initial condition $u(x, 0)=\exp \left(-x^{2}\right)$ and $v(x, 0)=0.2 \exp \left(-(x+2)^{2}\right)$ and $x \in[-\pi, \pi]($ left: $u ;$ right: $v)$

and

$$
\partial_{t} v(x, t)=-\frac{1}{\epsilon} u^{2}(x, t) v(x, t)
$$

The convergence result for an order $p$ splitting applied to this system can readily be seen to be the same as Theorem 1 . However, the constants in the estimates (3.3)-(3.5) depend on the small parameter $\epsilon, C=C\left(M_{2 p}, \epsilon^{-p}\right)$ in (3.3), $C=$ $C\left(M_{2 p-1}, \epsilon^{1-p}\right)$ in (3.4), and $C=C\left(M_{2 p-2}, \epsilon^{2-p}\right)$ in (3.5). We must stress that the involved estimates of the exact solution will also be negatively influenced when $\epsilon$ is small. The analysis of the exact solution is not a topic of the present paper, however.

For our comparisons, we solve the problem in one spatial dimension, with $x \in$ $[-\pi, \pi]$, and choose $\epsilon=10^{-3}$. The evolution of the solution components with $t$ (on the vertical axis) is illustrated in Fig. 7. Results showing the effectiveness of adaptive time-stepping for (4.1a) are shown in Table 2. For this problem, the lowerorder method is more efficient. Adaptive step selection yields a speed-up by about a factor 5. Indeed, if we consider the ratio of two consecutive step-sizes, we see some

Table 2 Comparison of the efficiency of [15, PP 5/6 A c] and [15, PP $3 / 4 \mathrm{~A} \mathrm{c}$ ] for (4.1a) with $D_{u}=$ $D_{v}=1$ and $\epsilon=10^{-3}, 256$ grid points

\begin{tabular}{lllll}
\hline Method & \# Steps adaptive & \# Steps equidist & Time adaptive & Time equidist \\
\hline PP 5 / 6 A c, tol $=10^{-3}$ & 11886 & 127118 & $6.41 \mathrm{e}+03$ & $3.14 \mathrm{e}+04$ \\
PP 3 / 4 A c, tol $=10^{-3}$ & 20989 & 217760 & $4.32 \mathrm{e}+03$ & $2.09 \mathrm{e}+04$ \\
& & & & \\
PP 5 / 6 A c, tol $=10^{-5}$ & 124559 & 1269018 & $6.20 \mathrm{e}+04$ & $3.13 \mathrm{e}+05$ \\
PP 3 / 4 A c, tol $=10^{-5}$ & 214338 & 2176945 & $3.81 \mathrm{e}+04$ & $2.09 \mathrm{e}+05$ \\
\hline
\end{tabular}

The final time was 10.0, with initial condition $u(x, 0)=\exp \left(-x^{2}\right)$ and $v(x, 0)=0.2 \exp \left(-(x+2)^{2}\right)$, and $x \in[-\pi, \pi]$ 


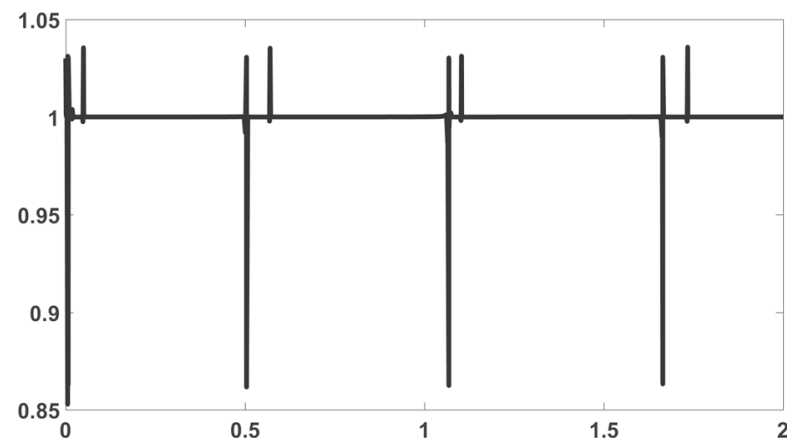

Fig. 8 Ratio of two consecutive time-step sizes for the solution of (4.1a) by [15, PP 5/6 A c]

variation in the region of the steep layers in Fig. 8, which is obviously sufficiently large to warrant adaptive time-stepping.

The time-steps generated in the course of an adaptive procedure are given in Fig. 9. The left plot shows the time-steps to satisfy a tolerance of $10^{-5}$ for the PP $3 / 4 \mathrm{~A}$ C method, and likewise on the right for the $[15, \mathrm{PP} 5 / 6 \mathrm{~A} \quad \mathrm{c}]$ pair.
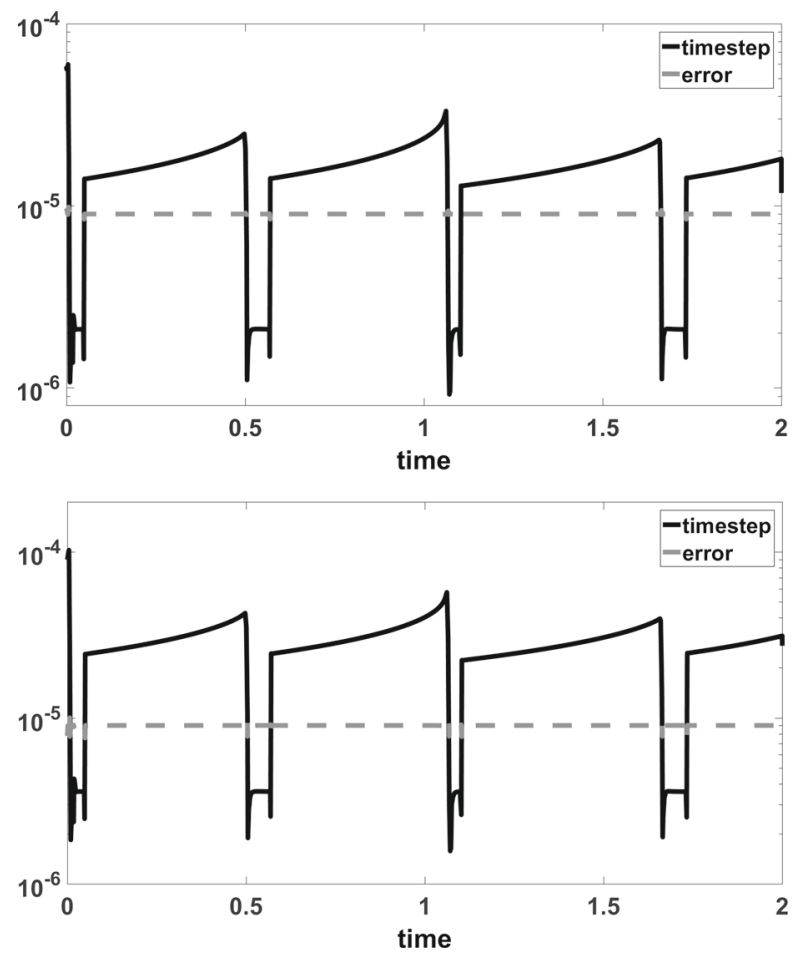

Fig. 9 Time-steps for (4.1a) for $[15$, PP $3 / 4$ A $\quad$ c $]$ (top), $\left[\begin{array}{lllll}15, \text { PP } & 5 / 6 & \text { A c c }\end{array}\right]$ (bottom), tolerance $10^{-5}$ 


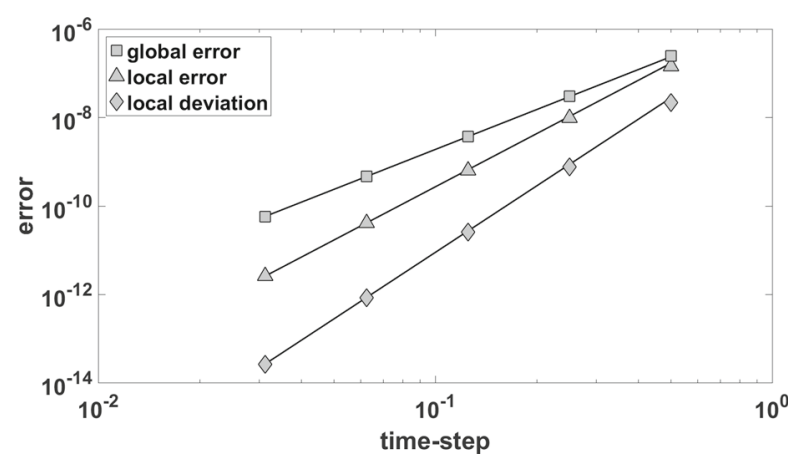

Fig. 10 Empirical convergence orders of the local and the global errors for the [15, PP $3 / 4$ A 3 c] splitting applied to the Gray-Scott (3)

\section{Splitting into three operators (“ABC-splitting”)}

Finally, we consider a splitting of the Gray-Scott (3) into three parts,

$$
\begin{aligned}
\underbrace{\left(\begin{array}{cc}
c_{1} \Delta-\alpha & 0 \\
0 & c_{2} \Delta-\beta
\end{array}\right) U(x, y, t)+\left(\begin{array}{l}
\alpha \\
0
\end{array}\right)}_{=A}+\underbrace{\left(\begin{array}{c}
0 \\
u(x, y, t) v^{2}(x, y, t)
\end{array}\right)}_{=B} \\
-\underbrace{\left(\begin{array}{c}
u(x, y, t) v^{2}(x, y, t) \\
0
\end{array}\right)}_{=C} .
\end{aligned}
$$

This has the computational advantage that the flows of the operators $B$ and $C$ can be computed analytically when the other component is frozen. Below, we verify the convergence orders for this case for the optimal palindromic splitting PP $3 / 4$ A 3 C.

Remark A formal error analysis for $A B C$-splitting has not yet been given in the nonlinear case; the linear case has been treated in [13]. However, inspection of the commutators that would critically influence the error shows that a convergence result analogous to Theorem 1 will hold, since commutators of $B$ and $C$ vanish.

\subsection{Numerical results}

The numerical results below were computed by the method [15, PP $3 / 4$ A 3 c]. This is the method of order 3 with the smallest leading error coefficients (see [14]) we could determine and offers the advantage of the cheap error estimator from Section 2.2, see Fig. 10. 


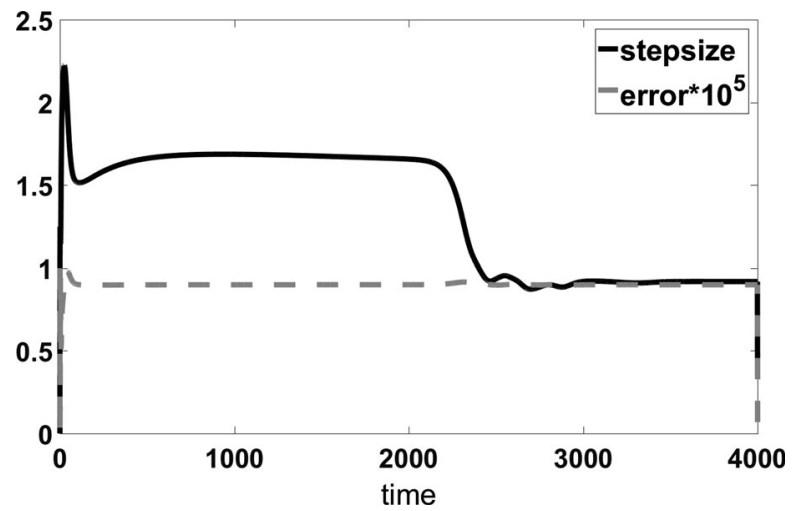

Fig. 11 Time-steps and local error for (3) for [15, PP $3 / 4$ A 3 c]

The time-steps generated in the course of an adaptive procedure according to Section 2.4 are given in Fig. 11. The plot shows the step-sizes to satisfy a tolerance of $10^{-5}$ for the PP $3 / 4$ A 3 C method.

\subsection{Comparisons}

In order to compare the efficiency of the $A B C$-splitting approach with the twooperator splitting discussed in Section 3, in Table 3, we give the number of steps required for tolerances $10^{-5}$ and $10^{-8}$ and the resulting computation times. It is observed that the $A B C$-splitting [15, PP $3 / 4$ A 3 C] requires slightly fewer steps than $[15, \mathrm{PP} 3 / 4 \mathrm{AC}$, but the computation time is higher. The reason is that each individual step is computationally more demanding in the $A B C$-splitting due to the larger number of required FFT transforms associated with the larger number of compositions. These result from the fact that the number of order conditions is larger in the $A B C$ case and therefore, more free parameters are necessary to construct highorder methods. Indeed, 1000 steps with $[15, \mathrm{PP} 3 / 4 \mathrm{~A} \mathrm{C}$ ] required $110.75 \mathrm{~s}$, for [15, PP $3 / 4$ A 3 C] the timing was 199.95. However, we stress again that a major advantage of the $A B C$-splitting approach for this example lies in the fact that the computations of the nonlinear flows can resort to analytical solutions instead of numerical approximations as in Section 3.

Table 3 Efficiency of the [15, PP 3 / 4 A 3 c] splitting for (3)

\begin{tabular}{lllll}
\hline Method & \# Steps adaptive & \# Steps equidist & Time adaptive & Time equidist \\
\hline PP 3 / 4 A 3 c, tol $=10^{-5}$ & 65 & 67 & 26.74 & 13.23 \\
PP 3 / 4 A 3 c, tol $=10^{-8}$ & 555 & 645 & 244.41 & 138.38 \\
\hline
\end{tabular}

The tolerances were $10^{-5}$ (top) and $10^{-8}$ (bottom), respectively 


\section{Conclusions and outlook}

We have investigated high-order adaptive time-splitting methods for the solution of nonlinear evolution equations of parabolic type under periodic boundary conditions. The theoretical error analysis for the Gray-Scott equations and the Van der Pol equation shows the classical convergence orders under regularity assumptions on the exact solution implied by the Sobolev inequality for functions on the torus. The theory is illustrated by numerical computations showing the established convergence orders.

Moreover, adaptive time-stepping strategies have been demonstrated to improve both efficiency and reliability, where high-order methods generally yield a computational advantage for the approximation of regular solutions. Local error estimators based on embedded formulae of splitting coefficients are more efficient than estimators employing the adjoint method, but the former need to be constructed especially by a computationally demanding optimization procedure, while the latter principle can be applied invariantly for methods of odd order.

Indeed, it has been observed that for problems with rapidly varying solutions, an adaptive strategy yields an advantage as compared to uniformly using the smallest time-step required locally. Secondly, a good guess of the time-step size is not commonly available even when the solution is smooth, so adaptive adjustment saves from repeating runs until the optimal step-size is found.

Splitting into three operators promises a computational advantage for the calculation of the individual compositions, but the complexity of high-order integrators of this class implies a significant surplus of necessary compositions which negatively affects the performance.

Acknowledgments Open access funding provided by Austrian Science Fund (FWF). This work was supported by the Austrian Science Fund (FWF) under grant P24157-N13 and the Vienna Science and Technology Fund (WWTF) under the grant MA14-002. The computational results presented have been achieved in part using the Vienna Scientific Cluster (VSC). We would like to thank Benson K. Muite (University of Tartu, Estonia) for many helpful discussions and implementation of a first version of our numerical solver.

Open Access This article is distributed under the terms of the Creative Commons Attribution 4.0 International License (http://creativecommons.org/licenses/by/4.0/), which permits unrestricted use, distribution, and reproduction in any medium, provided you give appropriate credit to the original author(s) and the source, provide a link to the Creative Commons license, and indicate if changes were made.

\section{Appendix A: Periodic functions and their Fourier transforms}

In the following, we recapitulate material from [19] for the convenience of the reader. Consider

$$
\begin{aligned}
Q & =[-a, a]^{d} \quad \text { associated with the } d \text {-dimensional torus in } \mathbb{C}^{d}, \\
C^{n} & =\left\{u: Q \rightarrow \mathbb{C}, \quad u \in C^{n}(Q) \text { is a periodic function }\right\} .
\end{aligned}
$$

The space $L^{2}=L^{2}(Q)$ is a Hilbert space with the inner product

$$
\langle u, v\rangle_{L^{2}}=\int_{Q} \overline{u(x)} v(x) \mathrm{d} x .
$$


Fourier representation of $\boldsymbol{u} \in \boldsymbol{L}^{\mathbf{2}}$ Let $k=\left(k_{1}, \ldots, k_{d}\right) \in \mathbb{Z}^{d}$, and $|k|=\left|k_{1}\right|+$ $\cdots+\left|k_{d}\right|$.

Definition 1 The Fourier transform $\mathcal{F}: L^{2}(Q) \rightarrow \mathbb{Z}^{d}, u \mapsto \mathcal{F}(u)=\left(c_{k}\right)_{k \in \mathbb{Z}}$ is defined by

$$
c_{k}:=\frac{1}{(2 a)^{d}} \int_{Q} u(x) \mathrm{e}^{-\mathrm{i}(k \cdot x) / a} \mathrm{~d} x, \quad k \in \mathbb{Z}^{d},
$$

and the inverse transform yields the representation

$$
u(x)=\sum_{k \in \mathbb{Z}^{d}} c_{k} \mathrm{e}^{\mathrm{i} \pi(k \cdot x) / a} .
$$

Parseval's identity implies an isometric correspondence

$$
\|u\|_{L^{2}}=\left((2 a)^{d} \sum_{k \in \mathbb{Z}^{d}}\left|c_{k}\right|^{2}\right)^{\frac{1}{2}} .
$$

Remark 1 Since the torus has finite measure, we have $L^{q} \subseteq L^{p}$ for $1 \leq p \leq q \leq \infty$.

We introduce the following notations: $H^{s}=H^{s}(Q), \alpha=\left(\alpha_{1}, \ldots, \alpha_{d}\right) \in \mathbb{N}_{0}^{d}$, $|\alpha|=\alpha_{1}+\cdots+\alpha_{d}, \alpha !=\alpha_{1} ! \cdots \alpha_{d} !$. Weak derivatives are denoted by $D^{\alpha} u$. The norm on $H^{s}$ is

$$
\|u\|_{H^{s}}=\left(\sum_{|\alpha| \leq s}\left|D^{\alpha} u\right|^{2}\right)^{\frac{1}{2}} .
$$

$H^{s}$ is a Hilbert space with inner product

$$
\langle u, v\rangle_{H^{s}}=\sum_{|\alpha| \leq S}\left\langle D^{\alpha} u, D^{\alpha} v\right\rangle_{L^{2}} .
$$

Fourier representation of $\boldsymbol{D}^{\alpha} \boldsymbol{u}$ The weak derivative has the Fourier representation

$$
D^{\alpha} u(x)=\left(\frac{\mathrm{i} \pi}{a}\right)^{|\alpha|} \sum_{k \in \mathbb{Z}^{d}} k^{\alpha} c_{k} \mathrm{e}^{\mathrm{i} \pi(k \cdot x) / a},
$$

and thus

$$
\left|D^{\alpha} u(x)\right|^{2}=(2 a)^{d}\left(\frac{\pi}{a}\right)^{2|\alpha|} \sum_{k \in \mathbb{Z}^{d}} k^{2 \alpha}\left|c_{k}\right|^{2}
$$

as a consequence of Parseval's identity (6.1). Here, $k^{\alpha}=k_{1}^{\alpha_{1}} \cdots k_{d}^{\alpha_{d}}$.

In the following, we will need to resort to the fact that the norms on the Sobolev space $H^{s}$ can equivalently be stated in terms of the Fourier coefficients. The proof of the following lemma is given in [19]. 
Lemma 1 With computable constants $\underline{C}, \bar{C}$ depending on $d$ and $s$, we have

$$
\underline{C}\|u\|_{H^{s}} \leq\left((2 a)^{d} \sum_{k \in \mathbb{Z}^{d}}\left(1+|k|^{2 s}\right)\left|c_{k}\right|^{2}\right)^{\frac{1}{2}} \leq \bar{C}\|u\|_{H^{s}} .
$$

Lemma 1 shows that $H^{s}$ is identical to the space

$$
\left\{u(x)=\sum_{k \in \mathbb{Z}^{d}} c_{k} \mathrm{e}^{\mathrm{i} \pi(k \cdot x) / a} \in L^{2}, \quad \sum_{k \in \mathbb{Z}^{d}}\left(1+|k|^{2 s}\right)\left|c_{k}\right|^{2}<\infty\right\},
$$

and the norm $\|u\|_{H^{s}}$ is equivalent to the norm

$$
\|u\|_{H_{*}^{s}}=\left((2 a)^{d} \sum_{k \in \mathbb{Z}^{d}}\left(1+|k|^{2 s}\right)\left|c_{k}\right|^{2}\right)^{\frac{1}{2}} .
$$

Moreover, (6.2) serves as the definition of the spaces $H^{s}$ for non-integer $s$.

\section{Appendix B: Sobolev embeddings}

\section{Continuity}

Theorem 2 For $s>d / 2$, we have $H^{s} \subseteq C^{0}$, and the embedding $H^{s} \hookrightarrow C^{0}$ is continuous, i.e.,

$$
\|u\|_{\infty} \leq \mathcal{C}_{s}\|u\|_{H^{s}} \text { for all } u \in H^{s} .
$$

Proof The proof is indicated in [19]. In the following, we work out the argument in detail. Consider an arbitrary $u \in H^{s}$. With

$$
\begin{aligned}
& |u(x)|=\left|\sum_{k \in \mathbb{Z}^{d}} c_{k} \mathrm{e}^{\mathrm{i} \pi(k \cdot x) / a}\right| \leq \sum_{k \in \mathbb{Z}^{d}}\left|c_{k}\right|, \\
& \|u\|_{\infty} \leq \sum_{k \in \mathbb{Z}^{d}}\left|c_{k}\right|,
\end{aligned}
$$

the Cauchy-Schwarz inequality in $\ell^{2}=\ell_{d}^{2}$ yields

$$
\begin{aligned}
\|u\|_{\infty} & \leq \sum_{k \in \mathbb{Z}^{d}} \frac{1}{\left(1+|k|^{2 s}\right)^{\frac{1}{2}}}\left(1+|k|^{2 s}\right)^{\frac{1}{2}}\left|c_{k}\right| \\
& \leq\left(\sum_{k \in \mathbb{Z}^{d}} \frac{1}{1+|k|^{2 s}}\right)^{\frac{1}{2}} \cdot \underbrace{\left(\sum_{k \in \mathbb{Z}^{d}}\left(1+|k|^{2 s}\right)\left|c_{k}\right|^{2}\right)^{\frac{1}{2}}}_{=C\|u\|_{H_{*}^{s}}}
\end{aligned}
$$


(with $C=(2 a)^{-\frac{d}{2}}$ ), provided that the series

$$
\sum_{k \in \mathbb{Z}^{d}} \frac{1}{1+|k|^{2 s}}
$$

is convergent.

- $\quad$ For $d=1$,

$$
\sum_{k_{1}=-\infty}^{\infty} \frac{1}{1+\left|k_{1}\right|^{2 s}}=1+2 \sum_{k_{1}=1}^{\infty} \frac{1}{1+\left|k_{1}\right|^{2 s}}
$$

where

$$
\sum_{k_{1}=1}^{\infty} \frac{1}{1+\left|k_{1}\right|^{2 s}} \leq \sum_{k_{1}=1}^{\infty} \frac{1}{\left|k_{1}\right|^{2 s}}
$$

is convergent for $2 s>1$, i.e., $s>1 / 2=d / 2$.

- For general $d$, we consider

$$
\begin{aligned}
& \sum_{k \in \mathbb{Z}^{d}} \frac{1}{1+|k|^{2 s}} \leq C \sum_{k \in \mathbb{N}_{0}^{d}} \frac{1}{1+|k|^{2 s}}=\sum_{m=0}^{\infty} \sum_{\substack{k k \mid=m \\
k \in \mathbb{N}_{0}^{d}}} \frac{1}{1+m^{2 s}} \\
& =\sum_{m=0}^{\infty}\left(\begin{array}{c}
m+d-1 \\
d-1
\end{array}\right) \frac{1}{1+m^{2 s}} \leq C \sum_{m=0}^{\infty} \frac{m^{d}}{1+m^{2 s}}<\infty
\end{aligned}
$$

for $s>d / 2$.

This shows that, for $s>d / 2$, the series (6.4) is convergent and that $u \in H^{s}$ satisfies (6.3). Furthermore, the absolute summability of the Fourier coefficients $c_{k}$ implies that the Fourier series for $u$ is uniformly convergent, which in turn implies the continuity of $u$.

Corollary 1 For $s>d / 2+n$, we have $H^{s} \subseteq C^{n}$, and the embedding $H^{s} \hookrightarrow C^{n}$ is continuous, i.e.,

$$
\|u\|_{C^{n}} \leq \mathcal{C}_{s, n}\|u\|_{H^{s}} \quad \text { for all } u \in H^{s}
$$

\section{Integrability}

In order to study integrability properties of functions $u \in H^{s}$, we need to interrelate them to summability properties of its Fourier transform in $\ell^{q}$ spaces, with

$$
\|\hat{u}\|_{\ell q}=\left(\sum_{k \in \mathbb{Z}^{d}}\left|c_{k}\right|^{q}\right)^{\frac{1}{q}}
$$

$\left(\hat{u}=\left(u_{k}\right)_{k \in \mathbb{Z}^{d}}\right)$. For the proof of the following result, see [20, Theorem 2.1 and 2.2] and also [21]. 
Lemma 2 (Hausdorff-Young) Let $1 \leq p \leq 2$ and $\frac{1}{p}+\frac{1}{q}=1$. Then,

$$
\|\hat{u}\|_{\ell^{p}} \leq C\|u\|_{L^{q}},
$$

and

$$
\|u\|_{L^{q}} \leq C\|\hat{u}\|_{\ell^{p}} .
$$

Theorem 3 For $s<d / 2$ and

$$
2 \leq p<\frac{d}{\frac{d}{2}-s},
$$

we have $H^{s} \subseteq L^{p}$, and the embedding $H^{s} \hookrightarrow L^{p}$ is continuous, i.e.,

$$
\|u\|_{L^{p}} \leq C\|u\|_{H^{s}} \text { for all } u \in H^{s} .
$$

Remark 2 It can be shown that the assertion of Theorem 3 is also valid for the endpoint case $s=d / 2$ and $p<\infty$; see [19].

Proof The proof is indicated in [19]. In the following, we work out the argument in detail.

For $p=2$, the assertion is trivial. For $2<p<\infty$ and $\frac{1}{p}+\frac{1}{q}=1$, inequality (6.6) implies ${ }^{1}$

$$
\begin{aligned}
\|u\|_{L^{p}} & \leq C\|\hat{u}\|_{\ell^{q}}=C\left(\sum_{k \in \mathbb{Z}^{d}}\left|c_{k}\right|^{q}\right)^{\frac{1}{q}} \\
& =C\left(\sum_{k \in \mathbb{Z}^{d}}\left(\left(1+|k|^{2 s}\right)\left|c_{k}\right|^{2}\right)^{\frac{q}{2}}\left(1+|k|^{2 s}\right)^{-\frac{q}{2}}\right)^{\frac{1}{q}} \\
& \left.\left.\leq C\left[\sum_{k \in \mathbb{Z}^{d}}\left(1+|k|^{2 s}\right)\left|c_{k}\right|^{2}\right)^{\frac{q}{2}}\left(\sum_{k \in \mathbb{Z}^{d}}\left(1+|k|^{2 s}\right)^{-\frac{q}{2-q}}\right)^{\frac{2-q}{2}}\right]^{\frac{1}{q}}\right]^{\frac{2-q}{2 q}} \\
& =C\left(\sum_{k \in \mathbb{Z}^{d}}\left(1+|k|^{2 s}\right)\left|c_{k}\right|^{2}\right)^{\frac{1}{2}}\left(\sum_{k \in \mathbb{Z}^{d}}\left(1+|k|^{2 s}\right)^{-\frac{q}{2-q}}\right)^{\frac{2-q}{2 q}} \cdot \sum_{H^{s}}\left(\sum_{k \in \mathbb{Z}^{d}}\left(1+|k|^{2 s}\right)^{-\frac{q}{2-q}} \cdot\right.
\end{aligned}
$$

Here, we have used Hölder's inequality with conjugate exponents $\frac{2}{q}, \frac{2}{2-q}$, and Lemma 1. This estimate makes sense provided the sum in the latter expression is

\footnotetext{
${ }^{1}$ Here, $p$ plays the role of $q$ in (6.6) and vice versa. We have $1 \leq q \leq 2$.
} 
finite, i.e., if

$$
\sum_{k \in \mathbb{Z}^{d}}\left(\frac{1}{1+|k|^{2 s}}\right)^{\frac{q}{2-q}}=C \sum_{k \in \mathbb{N}_{0}^{d}}^{\infty}\left(\frac{1}{1+|k|^{2 s}}\right)^{\frac{q}{2-q}}<\infty .
$$

We reason as in the proof of Theorem 2: We have

$$
\begin{aligned}
& \sum_{k \in \mathbb{N}_{0}^{d}}\left(\frac{1}{1+|k|^{2 s}}\right)^{\frac{q}{2-q}}=\sum_{m=0}^{\infty} \sum_{\substack{|k|=m \\
k \in \mathbb{N}_{0}^{d}}}\left(\frac{1}{1+m^{2 s}}\right)^{\frac{q}{2-q}} \\
& =\sum_{m=0}^{\infty}\left(\begin{array}{c}
m+d-1 \\
d-1
\end{array}\right)\left(\frac{1}{1+m^{2 s}}\right)^{\frac{q}{2-q}} \leq C \sum_{m=0}^{\infty} \frac{m^{d}}{\left(1+m^{2 s}\right)^{\frac{2 s q}{2-q}}}<\infty
\end{aligned}
$$

for $(2 s q) /(2-q)>d$, i.e., $q>2 d /(2 s+d)$. With $1 / p+1 / q=1$, this is equivalent to $p<d /\left(\frac{d}{2}-s\right)$, as asserted.

In the special cases $d=1,2,3$, which are relevant to our analysis, this means:

$-\quad d=1$ : For $s<1 / 2$ and

we have $H^{s} \subseteq L^{p}$.

$$
2 \leq p<\frac{1}{\frac{1}{2}-s}
$$

$-\quad d=2$ : For $s<1$ and

$$
2 \leq p<\frac{2}{1-s}
$$

we have $H^{s} \subseteq L^{p}$. In particular, $H^{1} \subseteq L^{p}$ for all $1 \leq p<\infty$.

$-\quad d=3$ : For $s<3 / 2$ and

$$
2 \leq p<\frac{3}{\frac{3}{2}-s}
$$

we have $H^{s} \subseteq L^{p}$. In particular, $H^{1} \subseteq L^{6}$.

\section{References}

1. Söderlind, G., Wang, L.: Adaptive time-stepping and computational stability. J. Comput. Appl. Math. 185, 225-243 (2006)

2. Hairer, E., Lubich, C., Wanner, G.: Geometric numerical integration. Springer-Verlag, BerlinHeidelberg-New York (2002)

3. Hochbruck, M., Ostermann, A.: Exponential integrators. Acta Numer. 19, 209-286 (2010)

4. Descombes, S., Duarte, M., Dumont, T., Louvet, V., Massot, M.: Adaptive time splitting method for multi-scale evolutionary partial differential equations. Confluentes Math. 03, 413-443 (2011)

5. Jahnke, T., Lubich, C.: Error bounds for exponential operator splittings. BIT 40, 735-744 (2000)

6. Thalhammer, M.: High-order exponential operator splitting methods for time-dependent Schrödinger equations. SIAM J. Numer. Anal. 46(4), 2022-2038 (2008)

7. Lubich, C.: On splitting methods for Schrödinger-Poisson and cubic nonlinear Schrödinger equations. Math. Comp. 77, 2141-2153 (2008)

8. Koch, O., Neuhauser, C., Thalhammer, M.: Error analysis of high-order splitting methods for nonlinear evolutionary Schrödinger equations and application to the MCTDHF equations in electron dynamics. M2AN Math. Model. Numer. Anal. 47, 1265-1284 (2013) 
9. Blanes, S., Casas, F., Chartier, P., Murua, A.: Optimized high-order splitting methods for some classes of parabolic equations. Math. Comp. 82, 1559-1576 (2013)

10. Hansen, E., Ostermann, A.: Exponential splitting for unbounded operators. Math. Comp. 78, 14851496 (2009)

11. Auzinger, W., Koch, O., Thalhammer, M.: Defect-based local error estimators for splitting methods, with application to Schrödinger equations, Part II: Higher-order methods for linear problems. J. Comput. Appl. Math. 255, 384-403 (2013)

12. Koch, O., Neuhauser, C., Thalhammer, M.: Embedded split-step formulae for the time integration of nonlinear evolution equations. Appl. Numer. Math. 63, 14-24 (2013)

13. Auzinger, W., Koch, O., Thalhammer, M.: Defect-based local error estimators for high-order splitting methods involving three linear operators. Numer. Algorithms 70, 61-91 (2015)

14. Auzinger, W., Hofstätter, H., Ketcheson, D., Koch, O.: Practical splitting methods for the adaptive integration of nonlinear evolution equations. Part I: construction of optimized schemes and pairs of schemes, BIT Numer. Math., published online 28 July 2016

15. Auzinger, W., Koch, O.: Coefficients of various splitting methods, http://www.asc.tuwien.ac.at/ $\sim$ winfried/splitting/

16. Hairer, E., Nørsett, S., Wanner, G.: Solving ordinary differential equations I. Springer-Verlag, BerlinHeidelberg-New York (1987)

17. Press, W., Flannery, B., Teukolsky, S., Vetterling, W.: Numerical recipes in C - the art of scientific computing. Cambridge University Press, Cambridge (1988)

18. Gray, P., Scott, S.: Chemical waves and instabilities. Clarendon, Oxford (1990)

19. Robinson, J.: Infinite-dimensional dynamical systems. Cambridge University Press, Cambridge (2001)

20. Katznelson, Y.: An introduction to harmonic analysis. Dover Publications, Inc., New York (1968)

21. Rudin, W.: Real and complex analysis, 3rd edn. McGraw-Hill (1987) 